\title{
Comparison of Three Evolutionary Algorithms: GA, PSO, and DE
}

\author{
Voratas Kachitvichyanukul* \\ Industrial and Manufacturing Engineering, Asian Institute of Technology, Thailand
}

(Received June 29, 2012 / Revised: July 31, 2012 / Accepted: August 18, 2012)

\begin{abstract}
This paper focuses on three very similar evolutionary algorithms: genetic algorithm (GA), particle swarm optimization (PSO), and differential evolution (DE). While GA is more suitable for discrete optimization, PSO and DE are more natural for continuous optimization. The paper first gives a brief introduction to the three EA techniques to highlight the common computational procedures. The general observations on the similarities and differences among the three algorithms based on computational steps are discussed, contrasting the basic performances of algorithms. Summary of relevant literatures is given on job shop, flexible job shop, vehicle routing, location-allocation, and multimode resource constrained project scheduling problems.
\end{abstract}

Keywords: Evolutionary Algorithm, Genetic Algorithm, Particle Swarm Optimization, Differential Evolution

* Corresponding Author, E-mail: voratas@ait.ac.th

\section{INTRODUCTION}

Evolutionary methods for solving NP-hard optimization problems have become a very popular research topic in recent years. Among the many methods proposed, the three that are very similar and popular are the genetic algorithm (GA), particle swarm optimization (PSO), and differential evolution (DE). While GA is more well-established because of its much earlier introduction, the more recent PSO and DE algorithms have started to attract more attention especially for continuous optimization problems. While many papers were published based on these algorithms, most assessments are made empirically with specific cases on a particular application domain, and the two key performance indicators commonly used for comparison are the solution quality and solution time. Some example comparison studies include Hassan et al. (2005) for GA and PSO, Wisittipanich and Kachitvichyanukul $(2011,2012)$ for DE and PSO on job shop scheduling problems.

This paper attempts to make a general qualitative comparison of the three evolutionary algorithms based on two aspects of a good metaheuristic algorithm, i.e., diversification and intensification. The observation will be focused on the elements of operations required and their effects on the diversification and intensification capabilities of each algorithm.

The paper is organized as follows. A general evolutionary algorithm is described at the conceptual level to highlight the common elements in all the evolutionary algorithms. The three targeted evolutionary algorithms are then described in turn in subsequent sections. Similarities and differences among the algorithms are discussed and highlighted. Finally, some reviews of literature that contain direct comparison of the algorithms are summarized.

\section{GENERAL STEPS OF EVOLUTIONARY METHOD}

There are three main processes in all evolutionary algorithms. The first process is the initialization process where the initial population of individuals is randomly generated according to some solution representation. Each individual represents a solution, directly or indi- 
rectly. If an indirect representation is used, each individual must first be decoded into a solution. Each solution in the population is then evaluated for fitness value in the second process. The fitness values can be used to calculate the average population fitness or to rank the individual solution within the population for the purpose of selection. The third process is the generation of a new population by perturbation of solutions in the existing population. The three key processes are applied as shown in Figure 1. For a more recent in depth discussion of evolutionary algorithm, see Yu and Gen (2010).

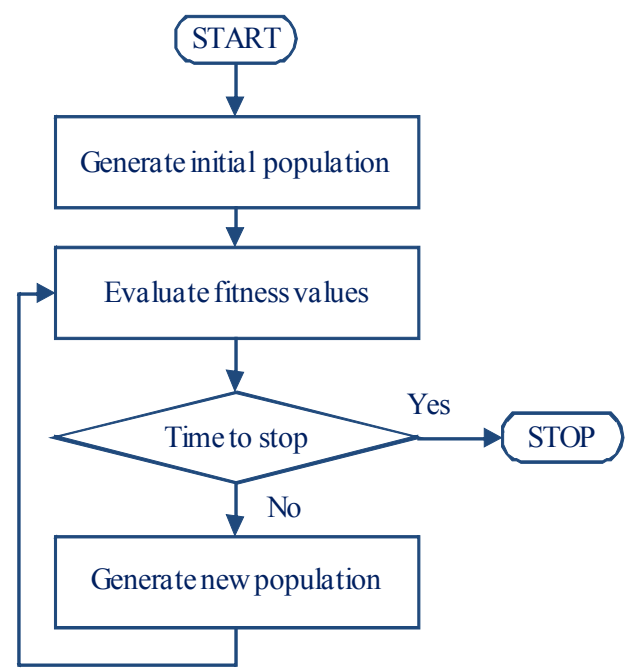

Figure 1. Flowchart of evolutionary algorithm.

As shown in Figure 1, after initialization, the population is evaluated and stopping criteria are checked. If none of the stopping criteria is met, a new population is generated again and the process is repeated until one or more of the stopping criteria are met. A stopping criterion may be static or dynamic. For example, a static stopping criterion may allow an algorithm to run for a fixed number of iterations. An example of a dynamic stopping criterion is to repeat the process until the top- $k$ percent of the solutions is within some percentage of the best solutions found. In some cases, a combination of several stopping criteria is used.

In using the evolutionary algorithm to solve optimization problems, the first important task is to determine how the solution can be represented according to the elements or terminology of the specific evolutionary algorithm. The processes for initialization and generation of new population may produce infeasible solutions. It is very important to choose a solution representation that is more likely to produce feasible solutions. This is a common design consideration for all evolutionary algorithms.

The solution representation can be a direct or an indirect one. The main design consideration is to ensure that each individual generated can always be decoded into a feasible solution. For a complex problem, indirect representation is often used along with a decoding pro- cedure to convert the indirect solution representation into a feasible solution. Once the solution is decoded, the fitness function can be evaluated.

In addition to the solution representation, two common parameters that must be determined initially are the population size and the maximum number of iteration. The choices of values of these two parameters have major influence on the solution quality and solution time, and in practice, these values are almost always determined empirically through pilot runs.

\section{GENETIC ALGORITHM}

Although GA started much earlier than 1975, Holland (1975) is the key literature that introduced GA to broader audiences. The flowchart of the genetic algorithm is given in Figure 2.

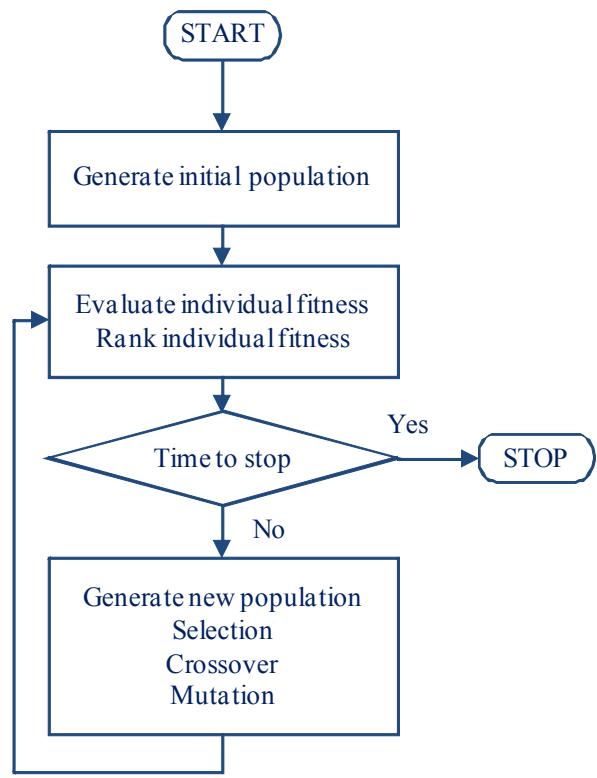

Figure 2. Flowchart for genetic algorithm.

The main idea of GA is to mimic the natural selection and the survival of the fittest. In GA, the solutions are represented as chromosomes. The chromosomes are evaluated for fitness values and they are ranked from best to worst based on fitness value. The process to produce new solutions in GA is mimicking the natural selection of living organisms, and this process is accomplished through repeated applications of three genetic operators: selection, crossover, and mutation. First, the better chromosomes are selected to become parents to produce new offspring (new chromosomes). To simulate the survivor of the fittest, the chromosomes with better fitness are selected with higher probabilities than the chromosomes with poorer fitness. The selection probabilities are usually defined using the relative ranking of the fitness values. Once the parent chromosomes are selected, the crossover operator combines the chromo- 
somes of the parents to produce new offspring (perturbation of old solutions). Since stronger (fitter) individuals are being selected more often, there is a tendency that the new solutions may become very similar after several generations, and the diversity of the population may decline; and this could lead to population stagnation. Mutation is a mechanism to inject diversity into the population to avoid stagnation. More detailed discussions can be found in the more classic reference by Holland (1975), Goldberg (1989) and the more recent reference by Gen and Cheng (1997), and Gen et al. (2008).

In addition to the population size and the maximum number of iterations, several decisions on parameters must be made for GA. The first set of decisions is the selection method and the probability assignment mechanism that is based on fitness. Different selection methods may require different mechanisms for probability assignments to ensure the balance of the diversity of the new population and the improvement of solutions. There are many proposed selection methods in literature, but the two most popular ones are the roulette wheel selection and tournament selection. Crossover method and crossover probability are the second set of decisions to be made. Many crossover methods are reported in literature since simple crossover methods have the tendency to produce infeasible or unusable chromosomes for many complex optimization problems. Finally, the mutation method and mutation probability must be selected as they may help to maintain the diversity of the population by injecting new elements into the chromosomes. In general, these three sets of decisions are set empirically using pilot runs.

There are many software implementations of GA available from various sources. A more general implementation of GA is the C++ GALib by Wall (1996) that allows the user to work at the source code level to apply GA with any representations and any genetic operators. The GALib classes provide the framework, and the user can solve a problem using GA by simply defining a representation, genetic operators, and objective function. Other software packages for GA are also available in EXCEL Solver (Frontline Solvers, http://www.solver.com/), and MatLab (http://www.mathworks.com/products/gads/).

\section{PARTICLE SWARM OPTIMIZATION}

In 1995, a paper on PSO was presented at the Congress on Evolutionary Computation (Kennedy and Eberhart, 1995). This landmark paper triggered waves of publications in the last decade on various successful applications of PSO to solve many difficult optimization problems. It is very appealing because of the simple conceptual framework and the analogy of birds flocking facilitated conceptual visualization of the search process. The basic PSO algorithm is shown in Figure 3.

In PSO, a solution is represented as a particle, and the population of solutions is called a swarm of particles.
Each particle has two main properties: position and velocity. Each particle moves to a new position using the velocity. Once a new position is reached, the best position of each particle and the best position of the swarm are updated as needed. The velocity of each particle is then adjusted based on the experiences of the particle. The process is repeated until a stopping criterion is met.

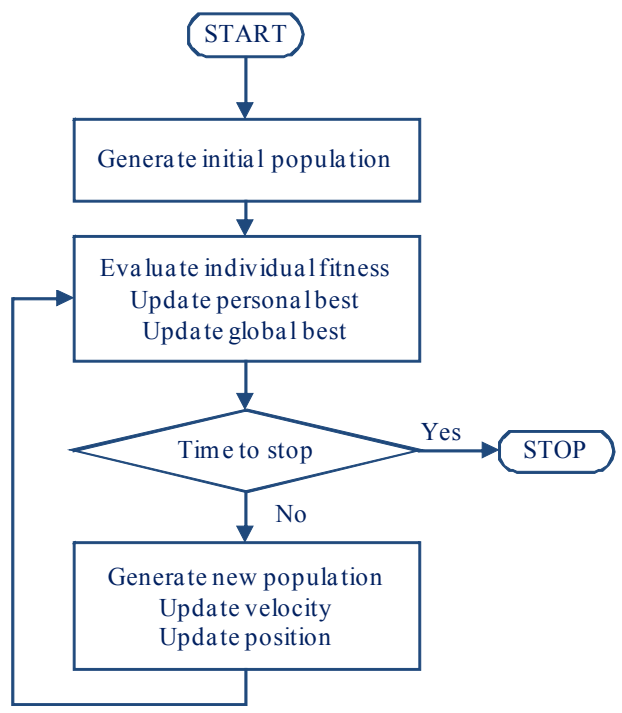

Figure 3. Flowchart for particle swarm optimization algorithm.

Similar to GA, the first process of PSO is initialization whereby the initial swarm of particles is generated. The concept of solution representation is also applied here in very much the same manner as GA. Each particle is initialized with a random position and velocity. Each particle is then evaluated for fitness value. Each time a fitness value is calculated, it is compared against the previous best fitness value of the particle and the previous best fitness value of the whole swarm, and the personal best and global best positions are updated where appropriate. If a stopping criterion is not met, the velocity and position are updated to create a new swarm. The personal best and global best positions, as well as the old velocity, are used in the velocity update.

As mentioned earlier, the two key operations in PSO are the update of velocity and the update of position. The velocity is updated based on three components: the old velocity (inertia or momentum term), experience of an individual particle (cognitive or self learning term), and experience of the whole swarm (group or social learning term). Each term has a weight constant associated with it. For basic PSO algorithm, the number of required constants is three.

It should be noted that PSO algorithm does not require sorting of fitness values of solutions in any process. This might be a significant computational advantage over GA, especially when the population size is large. The updates of velocity and position in PSO also only require a simple arithmetic operation of real numbers. 
Software implementation of PSO algorithm is now available in various forms, ranging from black-box to user modifiable source code. One software library for PSO algorithm that follows the design principle of GALib is named ET-Lib, Nguyen et al. (2010). The library is designed and implemented as object classes in C\# programming language, and the user must define the representation of particle and the objective function. Some successful applications of PSO that have utilized objects from ET-Lib include: Ai and Kachitvichyanukul (2009a, b, c, d), Nguyen and Kachitvichyanukul (2010), Pratchayaborirak and Kachitvichyanukul (2011), Kasemset and Kachitvichyanukul (2010, 2012), Sombuntham and Kachitvichyanukul (2010), and Sooksaksun et al. (2012).

\section{DIFFERENTIAL EVOLUTION}

DE was proposed about the same time as PSO by Storn and Price (1995) for global optimization over continuous search space. Its theoretical framework is simple and requires a relatively few control variables but performs well in convergence. For some unknown reason, DE caught on much slower than PSO but has lately been applied and shown its strengths in many application areas (Godfrey and Donald, 2006; Qian et al., 2008).

In $\mathrm{DE}$ algorithm, a solution is represented by a $\mathrm{D}-$ dimensional vector. DE starts with a randomly generated initial population of size $\mathrm{N}$ of D-dimensional vectors. In $\mathrm{DE}$, the values in the $\mathrm{D}$-dimensional space are commonly represented as real numbers. Again, the concept of solution representation is applied in DE in the same way as it is applied in GA and PSO.

The key difference of DE from GA or PSO is in a new mechanism for generating new solutions. DE generates a new solution by combining several solutions with the candidate solution. The population of solutions in DE evolves through repeated cycles of three main DE operators: mutation, crossover, and selection. However, the operators are not all exactly the same as those with the same names in GA.

The key process in $\mathrm{DE}$ is the generation of trial vector. Consider a candidate or target vector in a population of size $\mathrm{N}$ of D-dimensional vectors. The generation of a trial vector is accomplished by the mutation and crossover operations and can be summarized as follows. 1) Create a mutant vector by mutation of three randomly selected vectors. 2) Create trial vector by the crossover of mutant vector and target vector.

First, a mutant vector is generated by combining three randomly selected vectors from the population of vectors excluding the target vector. This combining process of three randomly selected vectors to form the mutant vector $V$ is defined as $V=X_{1}+F\left(X_{2}-X_{3}\right)$ where $X_{1}, X_{2}$, and $X_{3}$ are three randomly selected vectors from the population and $F$ is a multiplier which is the main parameter of the DE algorithm. The operation to form the mutant vector $V$ as described above is called muta- tion in DE, and this is unfortunate since the word "mutation" was used in GA much earlier with a totally different definition.

The second step is to create the trial vector by performing crossover between the mutant vector and the target vector. There are two commonly used crossover methods in DE: binomial crossover and exponential crossover. Here, the crossover probability must be specified. A small crossover probability leads to a trial vector that is more similar to the target vector while the opposite favors the mutant vector.

After the trial vector is formed for a given target vector, selection is done to keep only one of the two vectors. The simple criterion is to keep the vector with better fitness value. In other words, the target vector will survive if the trial vector has poorer fitness. Otherwise, the trial vector replaces the target vector immediately and becomes eligible for selection in the formation of the next mutant vector. This is an important difference since any improvement may affect other solutions without having to wait for the whole population to complete the update. The basic flow of a DE algorithm is summarized in Figure 4.

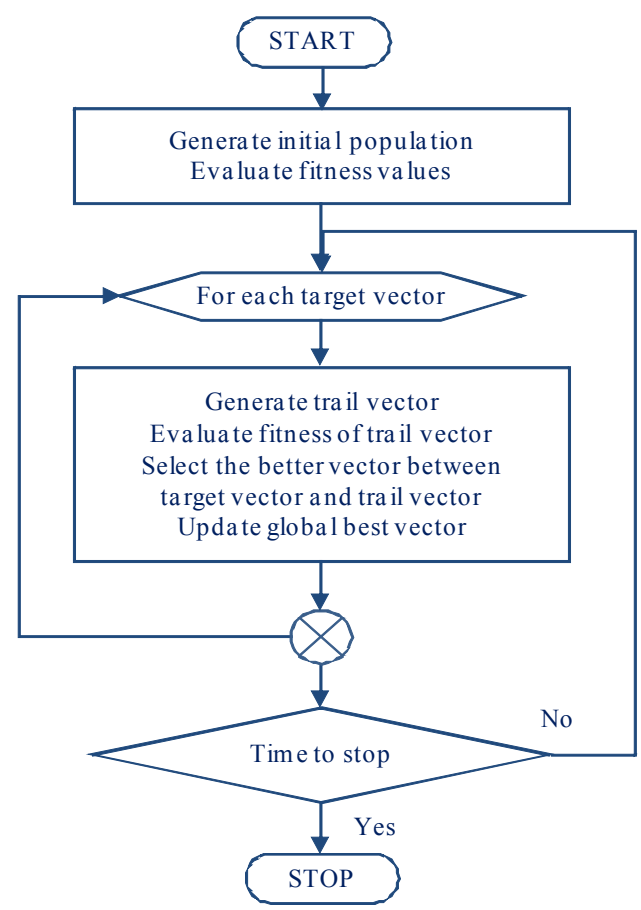

Figure 4. Flowchart for differential evolution.

As shown in Figure 4, the first process is the generation of a population of new solutions called vectors. Each vector in the population is evaluated for fitness value. Each vector takes turns as a candidate or target vector, and for each target vector, a trial vector is formed. The selection process simply chooses between the target vector and trial vector, i.e., the winning vector between the trial vector and the target vector survives into the next round while the losing vector is discarded. 
Several observations are made here. First, since a new solution would be selected only if it has better fitness, the average fitness of the population would be equal or better from iteration to iteration. Any improvement in the solution is immediately available to be randomly selected to form a mutant vector for the next target vector. This is different from GA and PSO where an improvement would take effect only after all the solutions has completed the iteration.

In contrast with GA where parent solutions are selected based on fitness, every solution in DE takes turns to be a target vector (one of the parents), and thus all vectors play a role as one of the parents with certainty. The second parent is the mutant vector which is formed from at least three different vectors. In other words, the trial vector is formed from at least four different vectors and would replace the target vector only if this new vector is better than the target vector; otherwise, it would be abandoned. This replacement takes place immediately without having to wait for the whole population to complete the iteration. This improved vector would then immediately be available for random selection of vectors to form the next mutant vector.

There are several variations of the DE proposed such as those including the best vector in the formation of the mutant vector or to use more vectors in the process. For more detailed information on the DE algorithm, see Price et al. (2005). Since the data element of the algorithm is very similar to PSO, a class library for DE was also developed using the same structure of ET-Lib.

\section{COMPARISON}

In the three algorithms discussed above, one of the key differences is in the mechanism to produce a new population of solutions via perturbation of solutions from the old population. These different mechanisms generate a population of solutions with different balance between intensification and diversification. This dynamic behavior of the population can be deducted from the basic perturbation method used in the creation of new solutions. This section discusses the three algorithms based on two aspects: intensification and diversification.

The discussion will be made algorithm by algorithm. Suppose that the same solution representation is used and the initial population is exactly the same. It should be noted that all evolutionary algorithms may require a decoding process and checking of constraints to ensure that the solutions are feasible.

For GA, the solutions are ranked based on the fitness values. The parents are selected based on probabilities that favor individuals with better fitness. The crossover operation produces offspring with parts taken from the parents and the solutions are more likely to be similar to the parents. Based on this observation, GA tends to generate solutions that are more likely to cluster around several "good" solutions in the population. The diversification aspect of GA is accomplished through the mutation operation that injects some "difference" into the solutions from time to time. The solution time of GA also increases non-linearly as the population size increases because of the required sorting.

For PSO, a new swarm of particles is generated via the velocity and position update equations. This ensures that all new particles can be much different than the old ones. Also, since the mechanism is based on the floating point arithmetic, it could generate any potential values within the solution space, i.e., the density of the solutions within the solution space may be much higher than those generated via GA. In other words, the solutions can be much closer to each other than solutions in GA. In addition, the best particle in the swarm exerts its oneway influence over all the remaining solutions in the population. This often leads to premature clustering around the best particle, especially if the fitness gaps are large.

Similar to PSO, since the mechanism to generate new solutions of $\mathrm{DE}$ is also based on the floating point arithmetic, the exploration ability of the population might be comparable to PSO, but the diversification is better because the best solution in the population does not exert any influence on the other solutions in the population. Furthermore, the mutant vector is always a solution that is not from the original population; therefore, the crossover operation in DE is always between a solution from the population and a newly generated one.

For any evolutionary algorithm, the solutions are gradually clustered around one or more "good" solutions as the search evolves. This clustering can be seen as the convergence of the population toward a particular solution. If the population clusters very quickly, the population may become stagnated and any further improvement becomes less likely.

\subsection{The Effect of Re-Initialization}

Among the three algorithms, PSO has a higher tendency to cluster rapidly and the swarm may quickly become stagnant. To remedy this drawback, several subgrouping approaches had been proposed to reduce the dominant influence of the global best particle. A much simpler and frequently used alternative is to simply keep the global best particle and regenerate all or part of the remaining particles. This has the effect of generating a new swarm but with the global best as one of the particles, and this process is called the re-initialization process. In GA, the clustering is less obvious, but it is often found that the top part of the population may look similar, and that re-initialization can also inject randomness into the population to improve the diversity. In DE, the clustering is the least and re-initialization has the least effect for DE.

\subsection{The Effect of Local Search}

In GA, the density of the population in the solution 
space is less, so it is often found that the GA operators cannot produce all potential solutions. A popular fix is the use of local search to see if a better solution can be found around the solutions produced by GA operators. The local search process is often time consuming, and to apply it over the whole population could lead to a long solution time. For PSO, the best particle has a dominant influence over the whole swarm, and a time saving strategy is to only apply local search to the best particle, and this can lead to solution improvement with shorter solution time. This strategy was demonstrated to be highly effective for job shop scheduling in Pratchayaborirak and Kachitvichyanukul (2011). This same strategy may not yield the same effect in DE since the best particle does not have a dominant influence on the population of solutions.

\subsection{The Effect of Sub-Grouping}

Sub-grouping is a simple strategy to delay premature clustering of solutions. Sub-grouping can be done either with homogeneous population or heterogeneous population. Homogeneous population refers to the fact that each solution in the population uses the same operators and the same parameters during the evolutionary process. Heterogeneous population allows solutions in different sub-group to use different operators and parameters, thus allow for more diverse search behavior. The use of sub-grouping of homogenous population to improve solution quality has been demonstrated in GA and PSO. This sub-grouping allows some groups of solutions to be freed from the influence of the dominant solutions, and thus the group may be searching in a different area of the solution space and improve the exploration aspect of the algorithms. For DE, the best particle has little influence on the perturbation process so it is rational to presume that sub-grouping with homogeneous population may have limited effect on the solution quality of DE. However, no research literature is found that addresses this issue. Pongchairerks and Kachitvichyanukul (2005) proposed a use of heterogeneous population in PSO to allow some fraction of the swarm to move by crossover with the best particle.

Dynamic change of population behavior can also achieve effects similar to the use of heterogeneous population. When more than one search strategies are included, the population can use the same search strategy as long as the solution continues to improve. If the solutions do not improve after some number of iterations, the population switches to use a different search strategy. Wisittipanich and Kachitvichyanukul (2012) applied strategy switching with DE for job shop scheduling problems.

\section{SUMMARY}

The comparisons made in earlier sections are tabulated in Table 1. There are many research literatures that compare performances of these three evolutionary algorithms in solving some difficult optimization problems in various domains. The comparisons are often made indirectly since many researchers applied different solution representations in combination with various local search. Thus it is not so clear if the contributor to the algorithm performance is from the evolutionary algorithm or from the local search. The comparison is more comprehensive when benchmark problems are used with the same solution representation and the same number of function evaluations. Some recent references on successful applications of evolutionary algorithms for important combinatorial problems are summarized in Table 2 .

\section{REFERENCES}

Ai, T. J. and Kachitvichyanukul, V. (2009a), A particle swarm optimization for the heterogeneous fleet vehicle routing problem, International Journal of Logistics and SCM Systems, 3(1), 32-39.

Ai, T. J. and Kachitvichyanukul, V. (2009b), A particle

Table 1. Qualitative comparison of GA, PSO, and DE

\begin{tabular}{|lccc|}
\hline & GA & PSO & DE \\
\hline \hline Require ranking of solutions & Yes & No & No \\
Influence of population size on solution time & Exponential & Linear & Linear \\
Influence of best solution on population & Medium & Most & Less \\
Average fitness cannot get worse & False & False & True \\
Tendency for premature convergence & Medium & High & Low \\
Continuity (density) of search space & Less & More & More \\
Ability to reach good solution without local search & Less & More & More \\
Homogeneous sub-grouping improves convergence & Yes & Yes & NA \\
\hline
\end{tabular}

GA: genetic algorithm, PSO: particle swarm optimization, DE: differential evolution. 
Table 2. List of relevant literatures for various domains of combinatorial optimization

\begin{tabular}{|c|c|}
\hline \multirow[t]{3}{*}{ Flowshop scheduling problem } & Kacem et al. (2002) \\
\hline & Liu, et al. $(2007 \mathrm{a}, \mathrm{b})$ \\
\hline & Pan et al. (2008) \\
\hline \multirow[t]{6}{*}{ Job shop scheduling problem } & Xia and $\mathrm{Wu}(2006)$ \\
\hline & Ge et al. (2008) \\
\hline & Pongchairerks and Kachitvichyanukul (2009) \\
\hline & Kachitvichyanukul and Sitthitham (2011) \\
\hline & Pratchayaborirak and Kachitvichyanukul (2011) \\
\hline & Wisittipanich and Kachitvichyanukul $(2011,2012)$ \\
\hline \multirow[t]{6}{*}{ Vehicle routing problem } & Hwang (2002) \\
\hline & Baker and Ayechew (2003) \\
\hline & Prins (2004) \\
\hline & Ai and Kachitvichyanukul (2009a, 2009b, 2009c, 2009d) \\
\hline & Marinakis and Marinaki (2010) \\
\hline & Sombuntham and Kachitvichyanukul (2010) \\
\hline \multirow[t]{3}{*}{ Flexible job shop problem } & Xia and $\mathrm{Wu}(2005)$ \\
\hline & Zhang and Gen (2005) \\
\hline & Pezzella et al. (2008) \\
\hline \multirow[t]{6}{*}{ Multicommodity distribution network design problem } & Canel et al. (2001) \\
\hline & Syarif et al. (2002) \\
\hline & Jaramillo et al. (2002) \\
\hline & Melo et al. (2005) \\
\hline & Amiri (2006) \\
\hline & Kachitvichyanukul et al. (2010) \\
\hline Multimode resource constrained project scheduling & Jarboui et al. (2008) \\
\hline \multirow[t]{3}{*}{ Problem } & Lova et al. (2009) \\
\hline & Van Peteghem and Vanhoucke (2010) \\
\hline & Nguyen and Kachitvichyanukul (2012) \\
\hline Warehouse design problem & Sooksaksun et al. (2012) \\
\hline \multirow[t]{2}{*}{ Bi-level job shop scheduling problem } & Qian et al. (2008) \\
\hline & Kasemset and Kachitvichyanukul $(2010,2012)$ \\
\hline
\end{tabular}

swarm optimization for vehicle routing problem with time windows, International Journal of Operational Research, 6(4), 519-537.

Ai, T. J. and Kachitvichyanukul, V. (2009c), A particle swarm optimization for the vehicle routing problem with simultaneous pickup and delivery, Computers and Operations Research, 36(5), 1693-1702.

Ai, T. J. and Kachitvichyanukul, V. (2009d), Particle swarm optimization and two solution representations for solving the capacitated vehicle routing problem, Computers and Industrial Engineering, 56(1), 380387.

Amiri, A. (2006), Designing a distribution network in a supply chain system: formulation and efficient solution procedure, European Journal of Operational Research, 171(2), 567-576.

Baker, B. M. and Ayechew, M. A. (2003), A genetic algorithm for the vehicle routing problem, Computers and Operations Research, 30(5), 787-800.

Canel, C., Khumawala, B. M., Law, J., and Loh, A. (2001), An algorithm for the capacitated, multi- commodity multi-period facility location problem, Computers and Operations Research, 28(5), 411427.

Ge, H.-W., Sun, L., Liang, Y.-C., and Qian, F. (2008), An effective PSO and AIS-based hybrid intelligent algorithm for job-shop scheduling, IEEE Transactions on Systems, Man and Cybernetics, Part A: Systems and Humans, 38(2), 358-368.

Gen, M. and Cheng, R. (1997), Genetic Algorithms and Engineering Design, Wiley, New York, NY.

Gen, M., Cheng, R., and Lin, L. (2008), Network Models and Optimization: Multiobjective Genetic Algorithm Approach, Springer, London, UK.

Godfrey, O. and Donald, D. (2006), Scheduling flow shops using differential evolution algorithm, European Journal of Operational Research, 171(2), 674-692.

Goldberg, D. E. (1989), Genetic Algorithms in Search, Optimization, and Machine Learning, Addison-Wesley Pub., Reading, MA.

Hassan, R., Cohanim, B., de Weck, O., and Venter, G. (2005), A comparison of particle swarm optimiza- 
tion and the genetic algorithm, Proceedings of the 1st AIAA Multidisciplinary Design Optimization Specialist Conference, Austin, TX.

Holland, J. H. (1975), Adaptation in Natural and Artificial Systems: An Introductory Analysis with Applications to Biology, Control and Artificial Intelligence, University of Michigan Press, Ann Arbor, MI.

Hwang, H.-S. (2002), An improved model for vehicle routing problem with time constraint based on genetic algorithm, Computers and Industrial Engineering, 42(2-4), 361-369.

Jaramillo, J. H., Bhadury, J., and Batta, R. (2002), On the use of genetic algorithms to solve location problems, Computers and Operations Research, 29(6), 761-779.

Jarboui, B., Damak, N., Sirry, P., and Rebai, A. (2008), A combinatorial particle swarm optimization for solving multi-mode resource-constrained project scheduling problems, Applied Mathematics and Computation, 195(1), 299-308.

Kacem, I., Hammadi, S., and Borne, P. (2002), Approach by localization and multiobjective evolutionary optimization for flexible job-shop scheduling problems, IEEE Transactions on Systems, Man, and Cybernetics, Part C: Applications and Reviews, 32(1), 1-13.

Kachitvichyanukul, V., Vinaipanit, M., and Kungwalsong, K. (2010), A genetic algorithm for multicommodity distribution network design of supply chain, International Journal of Logistics and Transport, 4(2), 167-181.

Kachitvichyanukul, V. and Sitthitham, S. (2011), A twostage genetic algorithm for multi-objective job shop scheduling problems, Journal of Intelligent Manufacturing, 22(3), 355-365.

Kasemset, C. and Kachitvichyanukul, V. (2010), Bi-level multi-objective mathematical model for job-shop scheduling: the application of theory of constraints, International Journal of Production Research, $\mathbf{4 8}$ (20), 6137-6154.

Kasemset, C. and Kachitvichyanukul, V. (2012), A PSObased procedure for a bi-level multi-objective TOCbased job-shop scheduling problem, International Journal of Operational Research, 14(1), 50-69.

Kennedy, J. and Eberhart, R. (1995), Particle swarm optimization, Proceedings of IEEE International Conference on Neural Networks, Perth, WA, 1942-1948.

Liu, B., Wang, L., and Jin, Y.-H. (2007a), An effective hybrid particle swarm optimization for no-wait flow shop scheduling, The International Journal of Advanced Manufacturing Technology, 31(9/10), 10011011.

Liu, B., Wang, L., and Jin, Y.-H. (2007b), An effective PSO-based memetic algorithm for flow shop sche- duling, IEEE Transactions on Systems, Man, and Cybernetics Part B, 37(1), 18-27.

Lova, A., Tormos, P., Cervantes, M., and Barber, F. (2009), An efficient hybrid genetic algorithm for scheduling projects with resource constraints and multiple execution modes, International Journal of Production Economics, 117(2), 302-316.

Marinakis, Y. and Marinaki, M. (2010), A hybrid genetic-particle swarm optimization algorithm for the vehicle routing problem, Expert Systems with Applications, 37(2), 1446-1455.

Melo, M. T., Nickel, S., and Saldanha de Gama, F. (2006), Dynamic multi-commodity capacitated facility location: a mathematical modeling framework for strategic supply chain planning, Computers and Operations Research, 33(1), 181-208.

Nguyen, S., Ai, T. J., and Kachitvichyanukul, V. (2010), Object Library for Evolutionary Techniques (ETLib): User's Manual, Asian Institute of Technology, Tailand.

Nguyen, S. and Kachitvichyanukul, V. (2010), Movement strategies for multi-objective particle swarm optimization, International Journal of Applied Metaheuristic Computing, 1(3), 59-79.

Nguyen, S. and Kachitvichyanukul, V. (2012), An efficient differential evolution algorithm for multimode resource constrained project scheduling problems, International Journal of Operational Research (Accepted March 2012).

Pan, Q.-K., Tasgetiren, M. F., and Liang, Y.-C. (2008), A discrete differential evolution algorithm for the permutation flowshop scheduling problem, Computers and Industrial Engineering, 55(4), 795-816.

Van Peteghem, V. and Vanhoucke, M. (2010), A genetic algorithm for the preemptive and non-preemptive multi-mode resource-constrained project scheduling problem, European Journal of Operational Research, 201(2), 409-418.

Pezzella, F., Morganti, G., and Ciaschetti, G. (2008), A genetic algorithm for the flexible job-shop scheduling problem, Computers and Operations Research, 35(10), 3202-3212.

Pongchairerks, P. and Kachitvichyanukul, V. (2005), A non-homogenous particle swarm optimization with multiple social structures, Proceedings of the International Conference on Simulation and Modeling, Bangkok, Thailand.

Pongchairerks, P. and Kachitvichyanukul, V. (2009), A two-level particle swarm optimisation algorithm on job-shop scheduling problems, International Journal of Operational Research, 4(4), 390-411.

Pratchayaborirak, T. and Kachitvichyanukul, V. (2011), A two-stage PSO algorithm for job shop scheduling problem, International Journal of Management Science and Engineering Management, 6(2), 84-93. 
Price, K. V., Storn, R. M., and Lampinen, J. A. (2005), Differential Evolution: A Practical Approach to Global Optimization, Springer, Berlin, Germany.

Prins, C. (2004) A simple and effective evolutionary algorithm for the vehicle routing problem, Computers and Operations Research, 31(12), 1985-2002.

Qian, B., Wang, L., Huang, D.-X., and Wang, W. (2008), Scheduling multi-objective job shops using a memetic algorithm based on differential evolution, The International Journal of Advanced Manufacturing Technology, 35(9-10), 1014-1027.

Sombuntham, P. and Kachitvichyanukul, V. (2010), Multi-depot vehicle routing problem with pickup and delivery requests, Proceedings of the International MultiConference of Engineers and Computer Scientists, Hong Kong, 71-85.

Sooksaksun, N., Kachitvichyanukul, V., and Gong, D.-C. (2012), A class-based storage warehouse design using a particle swarm optimisation algorithm, International Journal of Operational Research, 13(2), 219-237.

Storn, R. and Price, K. (1995), Differential evolution-a simple and efficient adaptive scheme for global optimization over continuous spaces, Technical Report TR-95-012, International Computer Science Institute, Berkeley, CA.

Syarif, A., Yun, Y., and Gen, M. (2002), Study on multi- stage logistic chain network: a spanning tree-based genetic algorithm approach, Computers and Industrial Engineering, 43(1/2), 299-314.

Wall, M. (1996), GAlib: A C++ library of genetic algorithm components, http://lancet.mit.edu/ga/.

Wisittipanich, W. and Kachitvichyanukul, V. (2011), Differential evolution algorithm for job shop scheduling problem, Industrial Engineering and Management Systems, 10(3), 203-208.

Wisittipanich, W. and Kachitvichyanukul, V. (2012), Two enhanced differential evolution algorithms for job shop scheduling problems, International Journal of Production Research, 50(10), 2757-2773.

Xia, W. and Wu, Z. (2005), An effective hybrid optimization approach for multi-objective flexible jobshop scheduling problems, Computers and Industrial Engineering, 48(2), 409-425.

Xia, W. and Wu, Z. (2006), A hybrid particle swarm optimization approach for the job-shop scheduling problem, The International Journal of Advanced Manufacturing Technology, 29(3/4), 360-366.

$\mathrm{Yu}, \mathrm{X}$. and Gen, M. (2010), Introduction to Evolutionary Algorithms, Springer, London, UK.

Zhang, H. and Gen, M. (2005), Multistage-based genetic algorithm for flexible job-shop scheduling problem, Journal of Complexity International, 11, 223-232. 